\title{
A Mixed-Integer Programming Approach to Interference Exploitation for Massive-MIMO
}

\author{
Pierluigi Vito Amadori ${ }^{1}$, Student Member, IEEE, and Christos Masouros ${ }^{2}$, Senior Member, IEEE \\ University College of London, UK \\ Email: uceepva@ucl.ac.uk ${ }^{1}$, chris.masouros@ieee.org ${ }^{2}$
}

\begin{abstract}
A novel low-complexity transmission scheme for Massive Multiuser Multi-Input Multi-Output (M-MU-MIMO) is proposed, where Transmit Antenna Selection (TAS) and beamforming are jointly performed to exploit multiuser interference. Two separate solutions to the deriving optimization problem are proposed: a mixed-integer programming approach that can optimally solve the TAS-beamforming problem and a heuristic convex approach, based on the assumption of matched filtering beamforming. Numerical results prove that the proposed multiuser interference exploiting approaches are able to greatly outperform previous state-of-the-art schemes, where TAS and beamforming are disjointedly solved.

Index Terms-Massive MIMO, Multiuser MIMO, Antenna selection, Convex Beamforming, Interference optimization
\end{abstract}

\section{INTRODUCTION}

Massive MIMO (M-MIMO) has experienced a growing interest from the research community [1] because of its promising benefits. More specifically, MIMO systems with very large arrays (VLAs) proved to be able to achieve extremely high throughputs and to be affected by very favorable propagation effects that lead linear precoding techniques to be asymptotically optimal [2]. At the same time, M-MIMO concepts can be directly applied to Multi-User (MU) scenarios, where the base station (BS) employs a VLA to perform secure, robust and energy-efficient communications with increased throughputs [3].

However, the practical implementation of M-MIMO comes with particularly challenging hardware and signal processing requirements. In fact, each radiating element needs a corresponding radio-frequency (RF) chain with its amplifier, analog-to-digital converter (ADC) and mixer. As a consequence, small active units [4] are expected to be preferred in order to respect cost and space constraints [5]-[7].

In the past, antenna selection [8] tackled the hardware complexity of multiple antenna systems by using only a subset of the available antennas. The selection can be performed according to several metrics, such as: received signal-to-noise ratio [9] and achievable capacity [10]. Recent works focused on antenna selection algorithms precisely designed for $\mathrm{M}$ MIMO systems, since a direct application to M-MIMO is nearly prohibitive [11]-[13]. More specifically, [14] and [15] proposed the use of convex optimization for M-MIMO AS systems, for a massively distributed antenna system and for channel capacity optimization, respectively.

Recently, concepts of constructive multi-user interference (MUI) have been applied to M-MU-MIMO, showing that the benefits of interference exploitation can be extended to large-scale systems. In fact, previous works [16]-[21] proved that downlink MUI can be manipulated in order to increase received power at the user side. Instead, the authors in [22] have extended interference-based symbol-level precoding concepts to MU-MIMO communications security applications via Directional Modulation [23].

Conventionally, transmit antenna selection (TAS) based schemes approach selection and downlink beamforming as two disjointed optimization problems, by first defining the antenna subset and then proceeding with linear or nonlinear precoding [8]-[12], [24]. In this paper, a novel transmission scheme is proposed, where both TAS and beamforming are jointly performed according to constructive interference exploitation. The deriving optimization is a Mixed-Integer Programming (MIP) problem and can be efficiently solved by commercial optimization solvers. In addition to the optimal MIP solution, a heuristic convex approach is proposed, where Matched Filter beamforming is assumed.

The proposed schemes are specifically designed for PSK modulation transmissions, however, recent works have shown that constructive MUI concepts can also be applied to Quadrature Amplitude Modulation (QAM). More specifically, the authors in [25], [26] have shown that the predictable interference at the BS can constructively superimpose with the desired signal at the receiver side for 16-QAM and [27] has provided constraints and metrics for constructive MUI in QAM and asymmetric phase-shift keying (APSK) modulations.

Notation: The following notation is used throughout the paper. Upper case boldfaced letters identify matrices (i.e. X), lower case boldfaced letters are used for vectors (i.e. $\mathbf{x}$ ), vector subindices are used to identify the columns of a matrix (i.e. $\mathbf{x}_{m}$ is the $m$-th column of $\left.\mathbf{X}\right)$, $\operatorname{diag}(\cdot)$ identifies the diagonal of a matrix, superscripts $(\cdot)^{T},(\cdot)^{H}$ and $(\cdot)^{*}$ stand respectively for transpose, Hermitian transpose and complex conjugate.

\section{SySTEM MODEL}

Consider a downlink transmission in a multiuser scenario, where the BS employs an $N$-sized VLA to communicate with $K$ single antenna users. The received signal $\mathbf{y}$ is a $\mathbb{C}^{K \times 1}$ vector, which collects the received complex symbols

$$
y_{k}=\mathbf{h}_{k}^{T} \mathbf{x}+n_{k}=\sum_{n=1}^{N} h_{n, k} x_{n}+n_{k},
$$


where $\mathbf{h}_{k}$ is the $\mathbb{C}^{N \times 1}$ channel vector experienced by the $k$ th user, i.e. the channel matrix $\mathbf{H}=\left[\mathbf{h}_{1}, \ldots, \mathbf{h}_{k}, \ldots, \mathbf{h}_{K}\right]$, and $n_{k}$ is the $k$-th component of the $\mathbb{C}^{K \times 1}$ zero mean additive white Gaussian noise vector $\mathbf{n}$, i.e. $\mathbf{n} \sim \mathcal{C N}\left(0, N_{0}\right)$ with $N_{0}$ being the noise variance. The entries of the channel matrix $\mathbf{H}$ represent the complex path gain between the $n$-th antenna at the BS and the $k$-th user and are modeled as independent Rayleigh fading, i.e. $h_{n, k}, \forall n \in\{1, \ldots, N\}, k \in\{1, \ldots, K\}$ is a zero mean independent and identically distributed complex Gaussian variable [28].

In a TAS-based system, the analytical definition of the $k$-th user received signal $\tilde{y}_{k}$ is modified as follows

$$
\tilde{y}_{k}=\sum_{n=1}^{N} h_{n, k} \tilde{x}_{n}+n_{k}
$$

where $\tilde{x}_{n}$ represents the $n$-th element of the $\mathbb{C}^{N \times 1}$ transmitted signal $\tilde{\mathbf{x}}$ and whose value is null when its index corresponds to one of the deactivated antennas, i.e., $\tilde{x}_{n}=0, \forall n \notin \mathcal{N}$ with $\mathcal{N}$ being the subset of transmitting antennas with cardinality $\operatorname{card}(\mathcal{N})=N_{t}$.

\section{A. Benchmark Schemes}

The proposed transmission schemes are compared to a cascade of existing state-of-the-art TAS and beamforming techniques. More specifically, two separate TAS algorithms are considered: a simple path gain-based selection [9], which has been thoroughly studied in the literature for its simplicity, and a capacity maximization technique [15], which can be performed through convex optimization and has shown to achieve near optimal performances. With regards to beamforming, both Signal-to-interference-and-noise ratio (SINR) balancing [29] and linear zero-forcing $(\mathrm{ZF})$ are considered.

1) Path Gain (PG) TAS: Path gain selection at the transmitter is easily performed by identifying the antennas with higher path gains [9]. Accordingly, the subset of transmitting antennas $\mathcal{N}$ can be analytically identified as

$\mathcal{N}=\arg \max _{N_{t}}\left\{\sum_{n=1}^{N}\left|h_{n, 1}\right|^{2}, \ldots, \sum_{n=1}^{N}\left|h_{n, k}\right|^{2}, \ldots, \sum_{n=1}^{N}\left|h_{n, K}\right|^{2}\right\}$

where max identifies the $N_{t}$ largest values of the argument.

2) Capacity (Cap) TAS: In [15], TAS is performed with the aim to maximize system sum-capacity under the assumption of dirty-paper coding (DPC) at the transmitter. System sumcapacity with DPC is defined as

$$
\mathcal{E}=\log _{2}\left[\operatorname{det}\left(\mathbf{I}_{K}+\rho \mathbf{H}^{H} \mathbf{\Delta} \mathbf{H}\right)\right]
$$

where $\mathbf{I}_{K}$ is a $K$-dimensional identity matrix, $\rho$ is the signalto-noise ratio and $\boldsymbol{\Delta}$ is the $N \times N$ dimensional selection matrix. The selection matrix is diagonal with binary entries: null values, i.e., $\Delta_{n, n}=0$, infer that the corresponding antennas are deactivated and unitary values, i.e., $\Delta_{n, n}=1$, identify the antennas that have been selected for transmission.
Accordingly, the optimization problem for sum-capacity maximization antenna selection can be defined as

$$
\begin{aligned}
& \mathcal{P}_{C a p}: \max _{\boldsymbol{\Delta}} \log _{2}\left[\operatorname{det}\left(\mathbf{I}_{K}+\rho \mathbf{H}^{H} \boldsymbol{\Delta} \mathbf{H}\right)\right] \\
& \text { s. t. } \sum_{n=1}^{N} \Delta_{n, n}=N_{t}, \Delta_{n, n} \in\{0,1\},
\end{aligned}
$$

The binary constraints imposed over the diagonal of the selection matrix cause the optimization problem $\mathcal{P}_{C a p}$ to be clearly non-convex. Nevertheless, such constraints can be relaxed and the deriving optimization problem was proven to be near-optimal when compared to exhaustive search approaches [15]. Accordingly, the convex-relaxation of $\mathcal{P}_{C a p}$ can be defined as

$$
\begin{array}{rll}
\mathcal{P}_{\text {Cap }}^{\prime}: & \max _{\Delta} & \log _{2}\left[\operatorname{det}\left(\mathbf{I}_{K}+\rho \mathbf{H}^{H} \mathbf{\Delta} \mathbf{H}\right)\right] \\
\text { s. t. } & \sum_{n=1}^{N} \Delta_{n, n}=N_{t}, \Delta_{n, n} \in\{0,1\},
\end{array}
$$

which leads to the TAS subset definition

$$
\mathcal{N}=\arg \max _{N_{t}}\left\{\Delta_{1,1}, \ldots, \Delta_{n, n}, \ldots, \Delta_{N, N}\right\} .
$$

3) SINR Balancing Beamformer: After the TAS is performed and the subset of transmitting antennas is identified, classical approaches from the literature proceed in defining the corresponding beamformer. In [29], a beamforming technique based on the maximization of the minimum received SINR is presented, here called SINR balancing. In fact, $k$-th user SINR is a direct function of the beamforming vectors $\mathbf{p}_{k}, \forall k$, as

$$
\gamma_{k}=\frac{\left|\mathbf{h}_{k}^{T} \mathbf{p}_{k}\right|^{2}}{\sum_{j \neq k}\left|\mathbf{h}_{k}^{T} \mathbf{p}_{j}\right|^{2}+N_{0}}=\frac{\left|\sum_{n=1}^{N} h_{n, k} p_{n, k}\right|^{2}}{\sum_{j \neq k}\left|\sum_{n=1}^{N} h_{n, k} p_{n, j}\right|^{2}+N_{0}},
$$

where $p_{n, k}$ represents the $n$-th element of the beamforming vector $\mathbf{p}_{k}$.

Accordingly, given a power constraint $P_{T}$ and a SINR threshold $\Gamma_{k}$, the SINR balancing optimization problem can be defined as

$$
\begin{aligned}
\mathcal{P}_{\text {SINR }}: & \max _{\mathbf{p}_{k}} \\
& \Gamma_{k} \\
\text { s.t. } & \gamma_{k} \geq \Gamma_{k}, \sum_{k=1}^{K}\left\|\mathbf{p}_{k}\right\|^{2} \leq P_{T},
\end{aligned}
$$

which is non-convex and requires an algorithmic approach in order to be solved [29].

After the weight-beamforming vectors of all the users have been identified, the transmitted signal is derived as $\mathbf{x}=\sum_{k=1}^{K} \mathbf{p}_{k} u_{k}$, where $u_{k}$ represents the constellation symbol corresponding to the $k$-th user.

\section{Constructive Interference Antenna SELECTION AND BEAMFORMING}

Multi-user interference (MUI) is generally considered as a damaging element for communications, with signal processing aiming to reduce its effects [29]-[31]. However, early works 


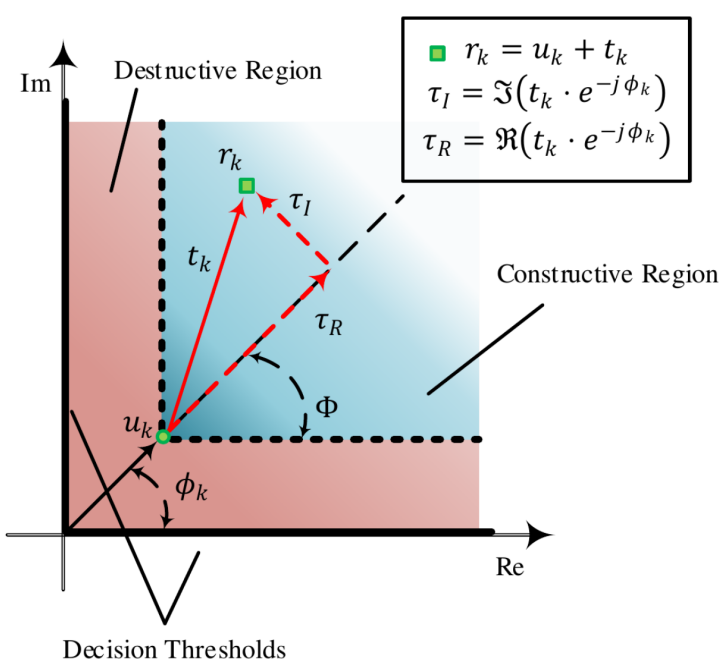

Fig. 1: Constructive and Destructive MUI regions for 4-PSK.

on interference exploitation for PSK signals have shown that both linear precoding [17] and TAS systems in [11] can benefit from MUI. In this section, the theoretical foundations of MUIexploitation in PSK modulations are first discussed and then applied to the proposed optimization problems for a joint TASbeamforming transmission.

\section{A. MUI exploitation in Phase-Shift-Keying (PSK) signals}

Multi-user interference symbols in PSK modulated transmissions can be deconstructed into the linear combination of a constructive or beneficial component and a destructive or detrimental component. The two components are distinguishable via simple geometrical concepts, described in details in MUI exploiting literature [17], [19], [20]. In general, constructive MUI causes the received signal in a noiseless scenario $r_{k}$ to be positioned further away from the decision thresholds of the desired constellation symbol $u_{k}$, while destructive MUI leads $r_{k}$ to lie closer to the decision thresholds. The MUI symbol for the $k$-th user can be explicitly defined in the following

$$
t_{k}=r_{k}-u_{k}=\sum_{n=1}^{N} h_{n, k} x_{n}-u_{k} .
$$

The condition for constructive MUI in a generalized $M$ ordered PSK modulation [19] is analytically expressed by the following inequality

$$
\Re\left(t_{k} \cdot e^{-j \phi_{k}}\right) \tan \Phi-\left|\Im\left(t_{k} \cdot e^{-j \phi_{k}}\right)\right| \geq 0,
$$

where $\Re(\cdot)$ and $\Im(\cdot)$ identify the extraction of the real and imaginary part of the complex argument, respectively, and $\Phi$ represents the central angle of the constellation sectors identified by the decision thresholds, whose value is a function of the constellation order $M$, i.e., $\Phi=\pi / M$. In (11), the MUI symbol $t_{k}$ is phase-shifted according to the phase of the desired constellation symbol for the $k$-th user $u_{k}=e^{j \phi_{k}}$, as it allows to isolate the effects of interference over phase and amplitude of $u_{k}$, as shown in Fig.1.

At the same time, the inequality (11) provides a quantitative evaluation of how constructive or destructive MUI is for the $k$-th user. In fact, a negative (11) implies that $r_{k}$ lies in the destructive region past the decision thresholds, the red area in Fig.1, while a positive (11) suggests that MUI is forcing $r_{k}$ further away from the decision thresholds, the blue region in Fig.1.

\section{B. Joint MIP Constructive Selection and Beamforming (MIP-} CSB)

The condition for constructive MUI in (11) can be used to define the following optimization problem, where TAS and beamforming are jointly performed

$$
\begin{aligned}
& \mathcal{P}_{C S B}: \quad \max _{\mathbf{a}, \tilde{\mathbf{x}}} \quad \min _{k}\left\{\Re\left(t_{k} e^{-j \phi_{k}}\right) \tan \Phi-\left|\Im\left(t_{k} e^{-j \phi_{k}}\right)\right|\right\} \\
& \text { s. t. } \quad t_{k}=\sum_{n=1}^{N} h_{n, k} \tilde{x}_{n}-u_{k}, \\
&\left|\tilde{x}_{n}\right| \leq a_{n}, \\
& \sum_{n=1}^{N} a_{n}=N_{t}, a_{n} \in\{0,1\} .
\end{aligned}
$$

Here, a represents the selection vector, whose entries are unitary when the corresponding antenna-index is connected to an RF chain for transmission and null when the corresponding antenna-index is deactivated. Binary constraints in (12) cause the optimization problem to be clearly non-convex, however it can be efficiently solved by commercial optimization tools such as MoSek. As we can see, $\mathcal{P}_{C S B}$ is designed in order to jointly perform the selection (i.e., by identifying the vector of transmitting antennas a) and design the transmitted signal $\tilde{\mathbf{x}}$. The joint optimization allows us to fully exploit the beneficial components of MUI, achieving significant transmission benefits and a particularly interesting trade-off between system complexity and performances.

\section{Joint Matched Filtering Constructive Selection (MFCS)}

In addition to the optimal solution achieved by MIP-CSB for joint TAS-beamforming, a heuristic approach to $\mathcal{P}_{C S B}$, called Matched Filtering Constructive Selection (MFCS), is proposed. In MFCS, the solution to TAS-beamforming is achieved by solving the convex optimization problem that derives under the key assumption that simple matched filtering (MF) beamforming is performed at the transmitter side. Accordingly, the optimization problem for TAS and beamforming can be represented as follows

$$
\begin{array}{cc}
\mathcal{P}_{M F C S}: & \max _{\tilde{\Delta}} \quad \min _{k}\left\{\Re\left(c_{k} e^{-j \phi_{k}}\right) \tan \Phi-\left|\Im\left(c_{k} e^{-j \phi_{k}}\right)\right|\right\} \\
\text { s. t. } & c_{k}=u_{k} \sum_{n=1}^{N} h_{n, k} \sum_{i=1}^{N} h_{i, k}^{*} \tilde{\Delta}_{i, n}-u_{k} \\
& \sum_{n=1}^{N} \tilde{\Delta}_{n, n}=N_{t}, \tilde{\Delta}_{n, n} \in[0,1] \\
& \tilde{\Delta}_{i, j}=0, \forall i \neq j .
\end{array}
$$


The optimization problem (13) is convex and can be efficiently solved by standard convex optimization tools. More specifically (13) can be represented as a standard SecondOrder Cone Programming (SOCP) problem [32] with a concave objective function [19]. In fact, the cost function of $\mathcal{P}_{M F C S}$ can be deconstructed into the combination of two functions: a linear function $\Re\left(c_{k} e^{-j \phi_{k}}\right)$ and a concave function $-\left|\Im\left(c_{k} e^{-j \phi_{k}}\right)\right|$, since the extraction of the imaginary and real part of a linear function preserves its linearity [19].

It is important to remark that (13) leads to a selection matrix $\tilde{\Delta}$ whose entries are not binary, since the binary constraints of $\mathcal{P}_{C S B}$ have been relaxed. Accordingly, before computing the selection vector $\mathbf{a}=\operatorname{diag}(\boldsymbol{\Delta})$, it is necessary to identify the binary counterpart of $\tilde{\Delta}$ as

$$
\Delta_{n, n}=\left\{\begin{array}{ccc}
1, & \forall & n \in\left\{\arg \max _{N_{t}}[\operatorname{diag}(\tilde{\boldsymbol{\Delta}})]\right\} \\
0 & \forall & n \notin\left\{\arg \max _{N_{t}}[\operatorname{diag}(\tilde{\boldsymbol{\Delta}})]\right\},
\end{array}\right.
$$

where $\arg \max _{N_{t}}[\cdot]$ is used to extract the indices of the $N_{t}$ largest elements of the argument.

After the selection vector $\mathbf{a}=\operatorname{diag}(\boldsymbol{\Delta})$ has been identified, the transmitted signal $\tilde{\mathbf{x}}$ can be computed as

$$
\tilde{x}_{n}= \begin{cases}1 / \xi_{n} \sum_{k=1}^{K} h_{n, k} u_{k}, & \text { if } \quad a_{n}=1, \\ 0 & \text { if } \quad a_{n}=0,\end{cases}
$$

where $\xi_{n}$ is a scaling factor, which guarantees a unitary transmitted power $\sum_{n=1}^{N}\left|\tilde{x}_{n}\right|^{2}=1$.

\section{RESULTS}

This section analyzes the proposed transmission schemes in terms of Symbol-Error-Rate (SER) at the receiver side, power efficiency and running times. Results are presented for 4-PSK transmissions; however, the proposed schemes can be directly applied to any PSK modulation order. Figure legends are characterized by the following notation: MIP-CSB identifies the MUI-exploiting scheme based the MIP solution, MFCS is used to classify the heuristic solution to the TASbeamforming problem based on the MF assumption at the BS and Cap-SINR represents the approach from the literature where TAS and beamforming are disjointly performed according to Cap selection [15] and SINR balancing [29], respectively. In addition, the proposed schemes are compared to two low-complexity approaches from the literature: PG$\mathrm{ZF}$, where path gain selection (PG) and zero forcing ( $\mathrm{ZF}$ ) linear beamforming are performed in cascade, and CIM-HY from [11], where TAS is performed in order to maximize constructive interference (CIM) and the transmitted signal is derived through hybrid (HY) linear precoding. In the proposed simulations, a single-cell downlink M-MIMO scenario with $K=5$ single-antenna mobile users is considered, where the BS possesses perfect channel-state information (CSI) and employs a VLA of $N=100$ antennas.

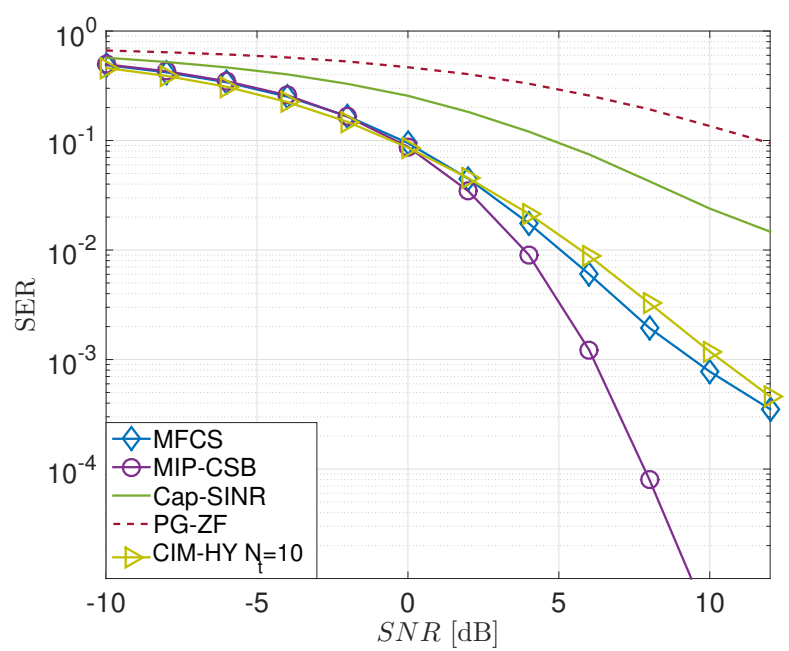

Fig. 2: 4-PSK Symbol Error Rate when $K=5, N=100$ and $N_{t}=5$ with perfect CSI.

\section{A. Symbol Error Rate (SER)}

Fig. 2 shows that the proposed schemes are able to rapidly achieve very low values of SER, while outperforming all the benchmark techniques, including the Cap-SINR scheme, characterized by a combination of Cap TAS [15] and SINR balancing beamforming [29]. While the MFCS scheme is based on a convex relaxation, it is still able to strongly outperform both the Cap-SINR and the PG-ZF techniques, hence proving to be a valuable approach for low-complexity and low-power scenarios. Additionally, it can be seen that the proposed schemes outperform the previous TAS scheme based on the exploitation of constructive MUI. The performance improvements are supported by two main factors: first, the optimality of the solutions achieved by the proposed MIP-CSB and MFCS schemes is guaranteed, and second, the proposed schemes perform a joint TAS-beamforming, which allows to achieve the maximum benefits from both problems, solved instead in a separate manner by CIM-HY.

\section{B. Power Efficiency}

The benefits and trade-offs introduced by the proposed schemes are further described by evaluating the power efficiency over throughput $\eta_{T}$, defined as the ratio

$$
\eta_{T}=\frac{T}{P_{a m p}+N_{t} \cdot P_{R F}}=\frac{(1-B L E R) \cdot m \cdot K}{P_{a m p}+N_{t} \cdot P_{R F}},
$$

where $B L E R$ is the block error rate, $m=\log _{2}(M)$ is the bit information per symbol, $K$ is the number of users in the chosen scenario, $P_{a m p}[W]$ represents the power consumption of the amplifier and $P_{R F}[W]$ identifies the power by one $\mathrm{RF}$ chain, which is characterized by digital-analog converter, mixer and filter. When computing (16), realistic power values from practical systems [33] are considered, where $P_{a m p}=$ $P_{t} / \nu$ is defined as the power required by an amplifier with $\nu=0.35$ efficiency and transmitted power $P_{t}=30 \mathrm{dBm}$ and 


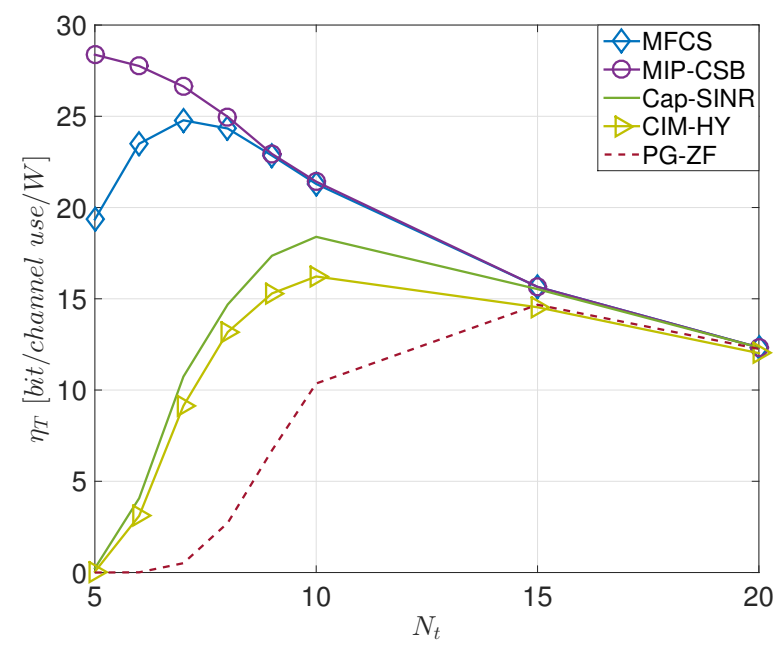

Fig. 3: 4-PSK Power Efficiency over Throughput when $K=5$, $N=100$ with perfect CSI and $S N R=5 d B$.

$P_{R F}=65.9 \mathrm{~mW}$. Power efficiency performances for the 4PSK case are presented in Fig. 3 as a function of the subset size $N_{t}$ when considering an $S N R=5 d B$.

Schemes from the literature are all outperformed by both MIP-CSB and MFCS for all the spectrum of subset size $N_{t}$ values. At the same time, it is interesting to notice that the proposed MFCS scheme is characterized by maximum value of $\eta_{T}$ when $N_{t}=7$, hence showing that 2 additional antennas in the subset size $N_{t}$ could further improve the SER performances shown in Fig. 2 with a beneficial trade-off between hardware complexity and power consumptions.

\section{Complexity Analysis}

The computational burdens of the proposed schemes are evaluated in terms of running times with increasing antenna array sizes at the BS. For the sake of a fair comparison, running times are computed within a coherence time, during which the channel state information $h_{n, k}, \forall n \in\{1, . ., N\}, k \in$ $\{1, . ., K\}$ is constant. This is due to the fact that the proposed schemes require to evaluate TAS-beamforming at a symbol-bysymbol rate, while the Cap TAS scheme needs to be performed on a coherence time basis. In the proposed study, a Time Division Duplexing (TDD) scenario [34] is considered, where coherence time $T_{\text {cohe }}$ represents the total number of datasymbols that can be transmitted while considering $\mathbf{H}$ constant. The TDD assumption is common on M-MIMO, as it allows to exploit the channel reciprocity property, which causes the number of slots used for CSI acquisition $T_{C S I}$ to be directly proportional to the number of users $K$. As it follows, the number of slots allocated to data transmission and reception can be computed as

$$
T_{\text {data }}=T_{\text {cohe }}-\mu K,
$$

where $\mu \geq 1$ is direct proportionality parameter that defines the number of pilot symbols used for CSI per user. Accord-

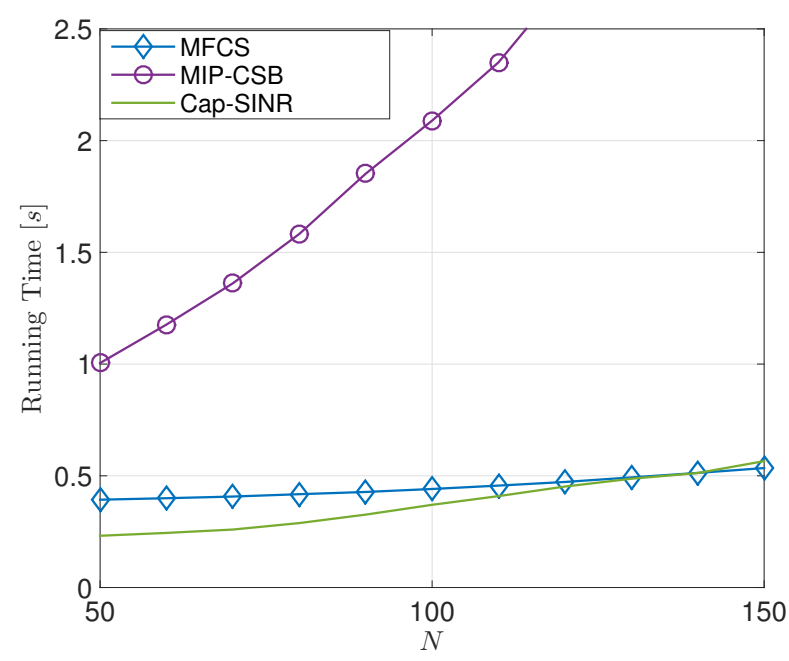

Fig. 4: Frame Running time when $K=5, N_{t}=5$ and $T_{D L}=$ 4.

ingly, the final number of data symbols to be allocated for downlink within a single coherence time is defined as

$$
T_{D L}=\eta_{D L}\left(T_{\text {cohe }}-T_{C S I}\right),
$$

where $\eta_{D L} \in[0,1]$ is the proportionality factor used to identify the downlink portion of $T_{\text {data }}$.

Running times are presented in Fig.4 for a realistic scenario where $T_{\text {cohe }}=10$ symbols, $T_{C S I}=5$ symbols and $T_{D L}=4$ symbols, in line with the work by [34]. It can be seen that the proposed schemes are overall affected by longer computational times. Such behavior is caused by the fact that the proposed schemes require a symbol-by-symbol update, in contrast with conventional TAS schemes from the literature. Nonetheless, the proposed MFCS scheme is characterized by running times that are comparable to the ones of Cap-SINR. This is particularly important, as it proves that such approach is able to achieve interesting performances in terms of SER and power efficiency with non-significant additional computational costs, when compared to Cap-SINR. The proposed MFCS scheme is almost unaffected by the increase in array sizes, while Cap-SINR is instead characterized by increasing running times for larger arrays. Accordingly, it can be inferred that the proposed MFCS scheme is expected to be characterized by similar or lower complexity for larger systems, when compared to existing state-of-the-art TAS schemes, such as Cap-SINR. On the other hand, the MIP-CSB curve shows that the MIP approach is characterized by higher computational times, which are caused by its trellis search-based solution.

However, Cap-SINR based schemes require further operations at the receiver in order to recover the data. In fact, the BS is required to feed-forward the mobile users with $\mathbf{h}_{k}^{T} \mathbf{p}_{k}, \forall k$ in order to equalize the received signal. On the contrary, such feedback is not required by the proposed approaches, where the computational complexity fully resides at the BS 
and additional operations at the receiver, such as estimation and equalization, are not necessary.

\section{CONClusions}

In this paper, transmit antenna selection and beamforming based on constructive MUI are jointly performed in order to improve power efficiency performances of future M-MUMIMO systems. The presented numerical studies show that constructive MUI at the receiver side can be efficiently optimized and exploited by simultaneously identifying a subset of transmitting antennas and the precoded signal. The proposed schemes are evaluated in terms of symbol error rate, power efficiency and computational complexity and their performances are compared with state-of-the-art schemes from the literature, showing the significantly positive trade-offs introduced by the proposed schemes.

\section{ACKNOWLEDGMENT}

This work was supported by the Royal Academy of Engineering, UK and the Engineering and Physical Sciences Research Council (EPSRC) project EP/M014150/1.

\section{REFERENCES}

[1] T. L. Marzetta, "Noncooperative cellular wireless with unlimited numbers of base station antennas," IEEE Transactions on Wireless Communications, vol. 9, no. 11, pp. 3590-3600, November 2010.

[2] F. Rusek, D. Persson, B. K. Lau, E. Larsson, T. Marzetta, O. Edfors and F. Tufvesson, "Scaling up MIMO: Opportunities and challenges with Very Large Arrays,” IEEE Signal Processing Magazine, vol. 30, no. 1, pp. 40-60, January 2013.

[3] E. Larsson, O. Edfors, F. Tufvesson, and T. L. Marzetta, "Massive MIMO for next generation wireless systems," IEEE Communications Magazine, vol. 52, no. 2, pp. 186-195, February 2014.

[4] A. Garcia-Rodriguez, V. Venkateswaran, P. Rulikowski, and C. Masouros, "Hybrid analog-digital precoding revisited under realistic RF modeling," IEEE Wireless Communications Letters, vol. 5, no. 5, pp. 528-531, October 2016.

[5] C. Masouros, M. Sellathurai, and T. Ratnarajah, "Large-Scale MIMO transmitters in fixed physical spaces: The effect of transmit correlation and mutual coupling," IEEE Transactions on Communications, vol. 61, no. 7, pp. 2794-2804, July 2013.

[6] C. Masouros and M. Matthaiou, "Space-Constrained Massive MIMO: Hitting the wall of favorable propagation," IEEE Communications Letters, vol. 19, no. 5, pp. 771-774, May 2015.

[7] S. Biswas, C. Masouros, and T. Ratnarajah, "Performance analysis of large multiuser MIMO systems with space-constrained 2-D antenna arrays," IEEE Transactions on Wireless Communications, vol. 15, no. 5, pp. 3492-3505, May 2016.

[8] S. Sanayei and A. Nosratinia, "Antenna selection in MIMO systems," IEEE Communications Magazine, vol. 42, no. 10, pp. 68-73, October 2004.

[9] Z. Chen, J. Yuan, B. Vucetic, and Z. Zhou, "Performance of Alamouti scheme with transmit antenna selection," Electronics Letters, vol. 39, no. 23, pp. 1666-1668, November 2003.

[10] A. Gorokhov, D. Gore, and A. Paulraj, "Receive antenna selection for MIMO spatial multiplexing: Theory and algorithms," IEEE Transactions on Signal Processing, vol. 51, no. 11, pp. 2796-2807, November 2003.

[11] P. V. Amadori and C. Masouros, "Interference driven antenna selection for massive multi-user MIMO," IEEE Transactions on Vehicular Technology, vol. 65, no. 8, pp. 5944-5958, August 2016.

[12] _ - "Large scale antenna selection and precoding for interference exploitation," IEEE Transactions on Communications, vol. 65, no. 10, pp. 4529-4542, October 2017.

[13] A. Garcia-Rodriguez, C. Masouros, and P. Rulikowski, "Reduced switching connectivity for large scale antenna selection," IEEE Transactions on Communications, vol. 65, no. 5, pp. 2250-2263, May 2017.
[14] S. Mahboob, R. Ruby, and V. Leung, "Transmit antenna selection for downlink transmission in a massively distributed antenna system using Convex Optimization," International Conference on Broadband, Wireless Computing, Communication and Applications (BWCCA), pp. 228-233, November 2012.

[15] X. Gao, O. Edfors, F. Tufvesson, and E. G. Larsson, "Massive MIMO in real propagation environments: Do all antennas contribute equally?" IEEE Transactions on Communications, vol. 63, no. 11, pp. 3917-3928, November 2015.

[16] E. Alsusa and C. Masouros, "Adaptive code allocation for interference management on the downlink of DS-CDMA systems," IEEE Transactions on Wireless Communications, vol. 7, no. 7, pp. 2420-2424, July 2008.

[17] C. Masouros, "Correlation rotation linear precoding for MIMO broadcast communications," IEEE Transactions on Signal Processing, vol. 59, no. 1, pp. 252-262, January 2011.

[18] C. Masouros, M. Sellathurai, and T. Ratnarajah, "Vector perturbation based on symbol scaling for limited feedback MISO downlinks," IEEE Transactions on Signal Processing, vol. 62, no. 3, pp. 562-571, February 2014.

[19] C. Masouros and G. Zheng, "Exploiting known interference as green signal power for downlink beamforming optimization," IEEE Transactions on Signal Processing, vol. 63, no. 14, pp. 3628-3640, July 2015.

[20] M. Alodeh, S. Chatzinotas, and B. Ottersten, "Constructive multiuser interference in symbol level precoding for the MISO downlink channel," IEEE Transactions on Signal Processing, vol. 63, no. 9, pp. 2239-2252, May 2015.

[21] — "Energy-Efficient symbol-level precoding in multiuser MISO based on relaxed detection region," IEEE Transactions on Wireles. Communications, vol. 15, no. 5, pp. 3755-3767, May 2016.

[22] A. Kalantari, M. Soltanalian, S. Maleki, S. Chatzinotas, and B. Ottersten, "Directional Modulation via symbol-level precoding: A way to enhance security," IEEE Journal of Selected Topics in Signal Processing, vol. 10, no. 8, pp. 1478-1493, December 2016.

[23] M. P. Daly and J. T. Bernhard, "Directional modulation technique for phased arrays," IEEE Transactions on Antennas and Propagation, vol. 57, no. 9, September 2009.

[24] P. V. Amadori and C. Masouros, "Constant envelope precoding by interference exploitation in phase shift keying-modulated multiuser transmission," IEEE Transactions on Wireless Communications, vol. 16, no. 1, pp. 538-550, January 2017.

[25] D. Kwon, H. S. Kang, and D. K. Kim, "Robust interference exploitationbased precoding scheme with quantized csit," IEEE Communications Letters, vol. 20, no. 4, pp. 780-783, April 2016.

[26] A. Li and C. Masouros, "Exploiting constructive mutual coupling in P2P MIMO by analog-digital phase alignment," IEEE Transactions on Wireless Communications, vol. 16, no. 3, pp. 1948-1962, March 2017.

[27] M. Alodeh, S. Chatzinotas, and B. Ottersten, "Symbol-Level Multiuser MISO Precoding for Multi-level Adaptive Modulation: A Multicast View," IEEE Transactions on Wireless Communications, vol. 16, no. 8 pp. 5511-5524, August 2017.

[28] H. Yang and T. L. Marzetta, "Performance of conjugate and zeroforcing beamforming in Large-Scale Antenna systems," IEEE Journal on Selected Areas in Communications, vol. 31, no. 2, pp. 172-179, February 2013.

[29] M. Schubert and H. Boche, "Solution of the multiuser downlink beamforming problem with individual SINR constraints," IEEE Transactions on Vehicular Technology, vol. 53, no. 1, pp. 18-28, January 2004.

[30] M. Joham, W. Utschick, and J. Nossek, "Linear transmit processing in MIMO communications systems," IEEE Transactions on Signal Processing, vol. 53, no. 8, pp. 2700-2712, August 2005.

[31] S. Mohammed and E. Larsson, "Per-Antenna constant envelope precoding for large Multi-User MIMO systems," IEEE Transactions on Communications, vol. 61, no. 3, pp. 1059-1071, March 2013.

[32] S. Boyd and L. Vandenberghe, Convex Optimization. Cambridge University Press, 2010.

[33] D. Ha, K. Lee, and J. Kang, "Energy efficiency analysis with circuit power consumption in massive MIMO systems," IEEE 24th International Symposium on Personal Indoor and Mobile Radio Communications (PIMRC), pp. 938-942, September 2013

[34] T. L. Marzetta, "How much training is required for multiuser MIMO?' Asilomar Conference on Signals, Systems and Computers (ACSSC), pp. 359-363, October 2006. 